\title{
AL amyloidosis with non-amyloid forming monoclonal immunoglobulin deposition; a case mimicking $\mathrm{AHL}$ amyloidosis
}

\author{
Shun Manabe ${ }^{1,2,3^{*}}$, Chihiro Iwasaki ${ }^{2,3}$, Michiyasu Hatano ${ }^{2}$, Fuyuki Kametani ${ }^{4}$, Masahide Yazaki ${ }^{5}$, Kosaku Nitta ${ }^{3}$ \\ and Michio Nagata ${ }^{1}$
}

\begin{abstract}
Background: Immunoglobulin heavy-and-light-chain amyloidosis (AHL amyloidosis) is a newly established disease entity where both the immunoglobulin heavy-chain and light-chain compose amyloid fibrils. The immunoglobulins responsible for the amyloid fibrils are generally identified by immunostaining and/or laser microdissection-liquid chromatography-tandem mass spectrometry (LMD-LC-MS/MS). However, both techniques do not biochemically differentiate immunoglobulins that formed amyloid fibrils from non-responsible immunoglobulins.

Case presentation: We herein report a case of 67-year-old female patient with renal amyloidosis due to lymphoplasmacytic lymphoma secreting monoclonal immunoglobulin M (IgM)-kappa. Renal immunostaining monotypically positive for IgM-kappa and LMD-LC-MS/MS identification of mu heavy-chain and kappa light-chain were consistent with the diagnosis of AHL amyloidosis. In order to confirm that both the immunoglobulin heavy-chain and light-chain were forming amyloid fibrils, we performed LC-MS/MS of renal amyloid fibrils isolated by the traditional amyloid purification method. The additional LC-MS/MS identified kappa light-chain only without any heavy-chain component. These results were suggestive that amyloid fibrils were composed by kappa light-chain only and that the mu heavy-chain identified by immunostaining and LMD-LC-MS/MS was derived from the non-specific co-deposition of monoclonal IgM-kappa.

Conclusion: The case was AL amyloidosis with non-amyloid forming monoclonal immunoglobulin deposition. While immunostaining and LMD-LC-MS/MS are irreplaceable techniques to classify amyloidosis, confident exclusion of the present condition should be required to diagnose $\mathrm{AHL}$ amyloidosis.
\end{abstract}

Keywords: Immunoglobulin light-chain amyloidosis, Immunoglobulin heavy-and-light chain-amyloidosis, Amyloid purification, Laser microdissection, Mass spectrometry

\section{Background}

Amyloidosis is a group of diseases characterised by deposition of insoluble fibrils derived from various proteins [1]. Immunoglobulin related amyloidosis (Ig-amyloidosis) is one of the most prevalent renal amyloidosis and is classified into immunoglobulin light-chain (AL) amyloidosis, immunoglobulin heavy-chain (AH) amyloidosis, and, the recently established disease entity, immunoglobulin heavy-and-light-chain (AHL) amyloidosis [1]. Most of the

\footnotetext{
* Correspondence: s1430436@u.tsukuba.ac.jp; shunmn5711@gmail.com ${ }^{1}$ Kidney and Vascular Pathology, Faculty of Medicine, University of Tsukuba, 1-1-1 Tennodai, Tsukuba, Ibaraki 305-8575, Japan

²Department of Nephrology, Yokohama Rosai Hospital, Yokohama, Kanagawa, Japan

Full list of author information is available at the end of the article
}

renal Ig-amyloidosis is $\mathrm{AL}$ amyloidosis, whereas $\mathrm{AH}$ and AHL amyloidosis are rare entities [2,3].

The classification of renal Ig-amyloidosis is generally performed by immunostaining of immunoglobulin light-chains and heavy-chains following positive Congo red stain [1]. Recently, laser microdissection (LMD) of glomerular amyloid depositions combined with liquid chromatography-tandem mass spectrometry (LC-MS/MS) has enabled more accurate classification [1]. In large-scale studies classifying renal amyloidosis by immunostaining and LMD-LC-MS/MS [2, 3], the amyloidosis with monotypic immunoglobulin light-chain and heavy-chain deposition were described as AHL amyloidosis. Therefore, at present, amyloidosis with monotypic immunoglobulin

(c) The Author(s). 2018 Open Access This article is distributed under the terms of the Creative Commons Attribution 4.0 International License (http://creativecommons.org/licenses/by/4.0/), which permits unrestricted use, distribution, and 
light-chain and heavy-chain deposition, with greater than or equal heavy-chain amount, is diagnosed as AHL amyloidosis [3]. However, immunostaining and LMD-LC-MS/ MS do not biochemically differentiate immunoglobulins that formed amyloid fibrils from non-amyloid, just deposited, immunoglobulins $[4,5]$. At the same time, the intact immunoglobulins could co-deposit with amyloid fibrils [4-6] and be identified by immunostaining $[4,6]$ and LMD-LC-MS/MS $[4,5]$.

We herein report a case of renal Ig-amyloidosis due to lymphoplasmacytic lymphoma, with immunostaining and LMD-LC-MS/MS results consistent with previously reported AHL amyloidosis [2, 3]. We attempted to confirm that both the immunoglobulin light-chain and heavy-chain were forming amyloid fibrils by LC-MS/MS of renal amyloid fibrils isolated by traditional amyloid purification method [7]. The additional LC-MS/MS only identified immunoglobulin light-chains as the component of amyloid fibrils. This case demonstrated "AL amyloidosis with non-amyloid forming monoclonal immunoglobulin deposition" that might be diagnosed as AHL amyloidosis by immunostaining and LMD-LC-MS/MS.

\section{Case report}

\section{Clinical history and laboratory results}

A 67-year-old Japanese woman, taking daily oral cyclophosphamide against lymphoplasmacytic lymphoma for 4 years, was referred to the nephrology department because of recently developed nephrotic syndrome. The patient showed bilateral leg edema and weight gain. Laboratory data showed preserved kidney function (serum creatinine, $0.61 \mathrm{mg} / \mathrm{dL}$; estimated glomerular filtration rate, $73.7 \mathrm{ml} / \mathrm{min} / 1.73 \mathrm{~m}^{2}$ ) and hypoalbuminemia $(2.2 \mathrm{~g} / \mathrm{dL})$. The urinalysis was negative for occult blood and revealed a urinary total protein-to-creatinine ratio (UPCR) of $9.7 \mathrm{~g} / \mathrm{gCr}$. Serum immunoglobulin levels were IgG $466 \mathrm{mg} / \mathrm{dL}$, IgA $74 \mathrm{mg} / \mathrm{dL}$, and IgM $2789 \mathrm{mg} / \mathrm{dL}$, serum free light-chain levels were kappa-light chain $24.4 \mathrm{mg} / \mathrm{L}$ and lambda-light chain $15.8 \mathrm{mg} / \mathrm{L}$ (kappa-to-lambda serum free light-chain ratio of 1.54) and plasma electrophoresis revealed IgM-kappa monoclonal gammopathy. Renal biopsy was performed under suspicion of monoclonal gammopathy of renal significance [1].

\section{Renal biopsy findings and clinical follow-up}

The renal biopsy specimens contained 12 glomeruli without proliferative features, and the glomeruli revealed slight mesangial widening with amorphous deposits (Fig. 1a). Congo-red staining was positive in the mesangial area (Fig. 1b) and electron microscopic analysis showed non-branched fibrils (Fig. 1c) indicating renal amyloidosis. Electron microscopic analysis was negative for granular and amorphous deposits on the endothelial aspect of the glomerular basement membrane and outer aspect of the tubular basement membrane. Immunofluorescence staining was equally positive for IgM (mu heavy-chain, Fig. 1d) and kappa light-chains (Fig. 1e) and was negative for linear staining of tubular basement membrane. The immunostaining for the other immunoglobulins (Fig. 1f), complements, serum amyloid A, transthyretin, and fibrinogen yielded negative results. Therefore, IgM-kappa type renal
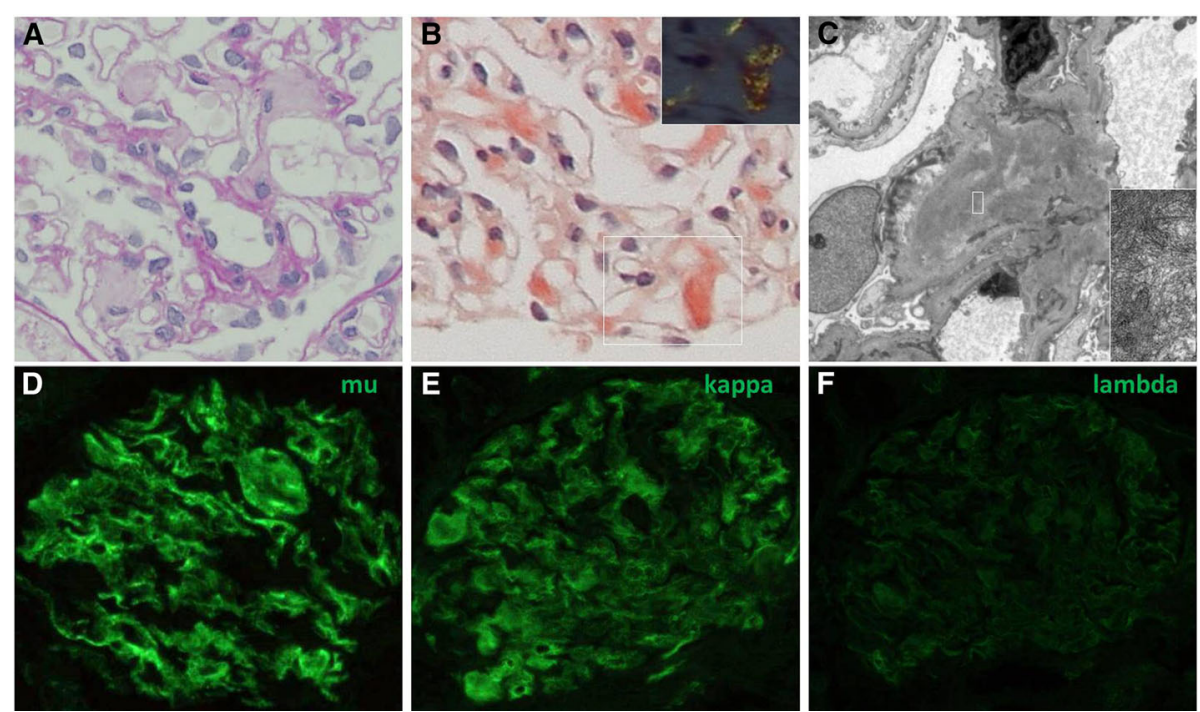

Fig. 1 Renal biopsy findings consistent with IgM-kappa type AHL amyloidosis. a Glomeruli with mesangial widening consisting amorphous deposition ( $\times 100$, periodic acid-Schiff stain), (b) Glomeruli with mesangial Congo red positive deposition $(\times 100)$, (c) Electron micrograph with mesangial organized deposition with fibrillary structure of 8 to $12 \mathrm{~nm}$ in diameter $(\times 1500, \times 20000)$, (d-f) Immunofluorescent staining positive for mu heavy-chain (d) and kappa light-chain (e) and negative for lambda light-chain (F) 
AHL amyloidosis due to lymphoplasmacytic lymphoma was suspected [1]. Smears and flow cytometry of bone marrow aspirate were consistent with previous diagnosis of lymphoplasmacytic lymphoma. After the diagnosis, weekly oral fludarabine was initiated. The proteinuria and hypoalbuminemia gradually improved to UPCR of $2.5 \mathrm{~g} / \mathrm{gCr}$ and serum albumin level of $3.6 \mathrm{~g} / \mathrm{dL}$ in one-and-a-half years.

\section{LC-MS/MS analyses}

In order to confirm the monotypic IgM-kappa deposition and the diagnosis of AHL amyloidosis, we performed LMD-LC-MS/MS of the glomerular amyloid depositions. The LMD-LC-MS/MS identified mu heavy-chain and kappa light-chain (Fig. 2a) with mu heavy-chain predominance consistent with the diagnosis of IgM-kappa type renal AHL amyloidosis [3]. We next performed LC-MS/ MS of renal amyloid fibrils isolated by the traditional amyloid purification method [7] to confirm that both the identified immunoglobulins compose amyloid fibrils. The additional LC-MS/MS only identified kappa light-chains without any heavy-chain component (Fig. 2b). All together, we eventually diagnosed the case as "kappa type renal AL amyloidosis with non-amyloid forming monoclonal IgM-kappa deposition".

\section{Discussion}

AHL amyloidosis is a disease entity where both the immunoglobulin light-chain and heavy-chain are composing amyloid fibrils, namely the concurrence of $\mathrm{AL}$ and AH amyloidosis $[1,4]$.

In $\mathrm{AL}$ amyloidosis, excess production of amyloidogenic light-chain lead to amyloid fibril formation [8]. In the present case, LMD-LC-MS/MS identified the peptide sequences of the kappa light-chain variable and constant domain (Fig. 2a), and the LC-MS/MS of isolated amyloid fibrils confirmed that variable domain were the component of the amyloid fibrils (Fig. 2b).

In contrast to AL amyloidosis, little is known about heavy-chain derived amyloid fibril formation. When reviewing the reported cases of $\mathrm{AH}$ amyloidosis (Table 1, $[2,3,9-17])$, it was presumable that amyloid fibrils are composed of the variable domains with or without

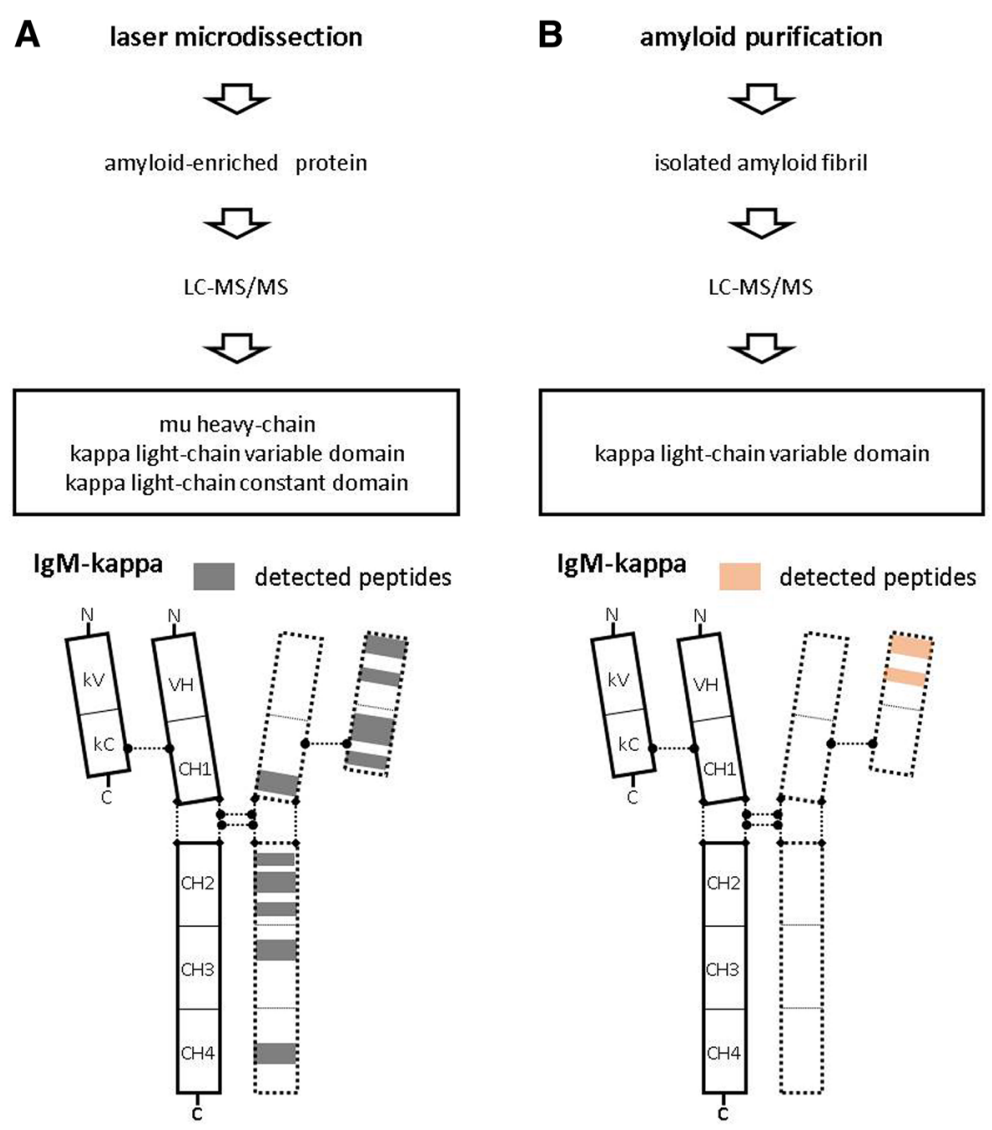

Fig. 2 Schematic diagrams of the two-LC-MS/MS analyses. a LMD-LC-MS/MS identified mu heavy-chain and kappa light-chain. The identified peptide sequences were identical to amino acid position 79-89, 121-128, 132-150, 164-177, 244-238, and 377-391 of mu heavy-chain, position 1-18 and 47-55 of kappa light-chain variable domain, and position 41-75 and 83-99 of kappa light-chain constant domain. b LC-MS/MS of isolated amyloid fibrils only identified kappa light-chain. The identified peptide sequences were identical to amino acid position 1-18 and 47-55 of kappa light-chain variable domain 


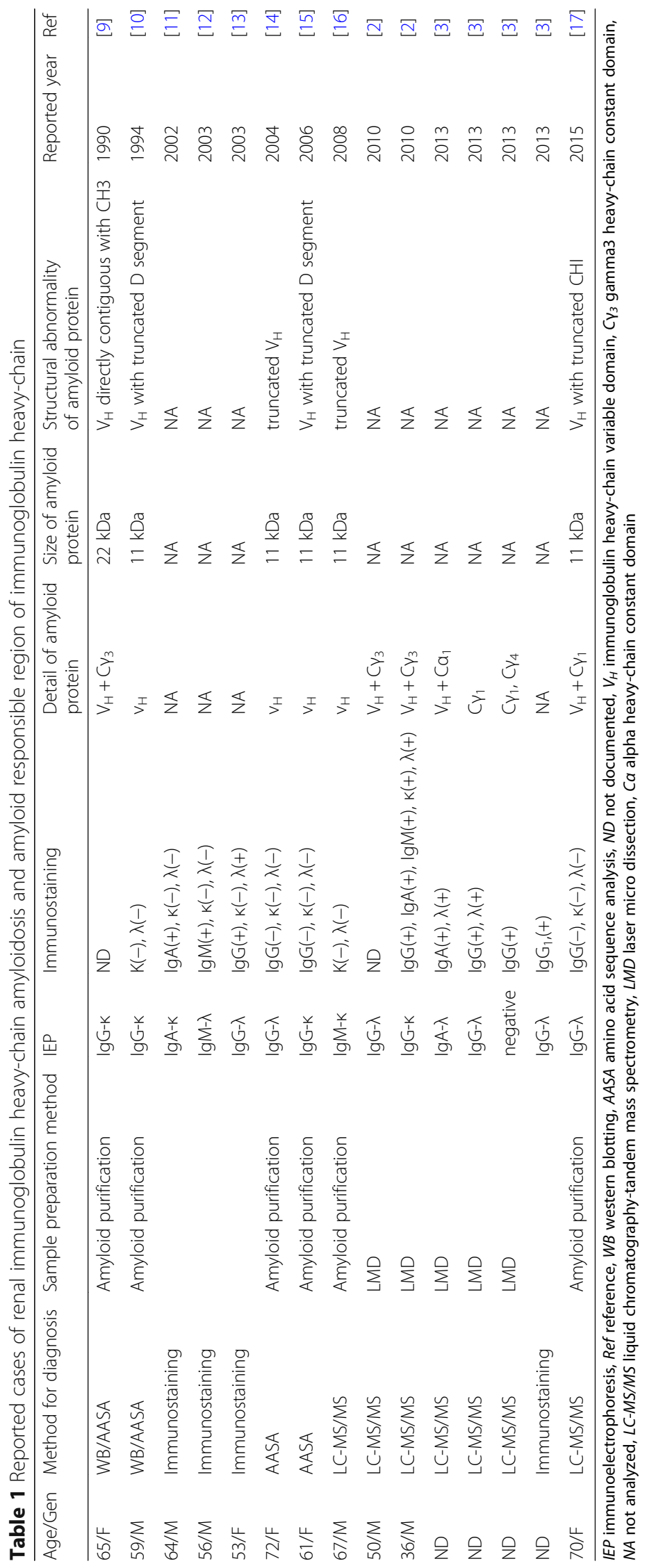


truncated small constant domains of immunoglobulin heavy-chain $[9,10,14-17]$. In the present case, LMD-LC-MS/MS identified peptide sequences that were broadly distributed among the constant domain $(\mathrm{CH} 1$, $\mathrm{CH} 2, \mathrm{CH} 3$, and $\mathrm{CH} 4$ domains) of $\mathrm{mu}$ heavy-chain (Fig. 2a) that do not match the previously reported $\mathrm{AH}$ amyloidosis cases. Therefore, the LMD-LC-MS/MS results were suggestive that the identified mu heavy-chain was not composing amyloid fibrils, and the LC-MS/MS of isolated amyloid fibrils confirmed the speculation (Fig. 2b).

There are several hypotheses to explain the glomerular $\mathrm{mu}$ heavy-chain deposition. First, there are reported cases of AL amyloidosis combined with non-amyloid monoclonal immunoglobulin deposition diseases (MIDDs) [18]. However, in the present case, there were no histological findings to support the concurrence of non-amyloid MIDD [1]. Second, concurrence of AL amyloidosis and heavy-chain disease cannot be denied [19]. However, plasma electrophoresis did not revealed free heavy-chain and smears and flow cytometry of the bone marrow aspirate were consistent with the lymphoplasmacytic lymphoma without detection of IgM positive light-chain negative lymphoplasmacytoid lymphocytes [20], Therefore, we speculate that the identified mu heavy-chain was derived from monoclonal IgM-kappa that non-specifically co-deposited with amyloid fibrils [5], while the exact origin remains unknown.

This case documented "AL amyloidosis with non-amyloid forming monoclonal immunoglobulin deposition" using immunostaining and two distinct LC-MS/MS analyses. In fact, the existence of the present condition has been speculated upon the initial description of AHL amyloidosis [4]. The concern was in part because of the technical limitations of immunostaining and LMD-LC-MS/ MS which do not differentiate amyloid fibrils from non-amyloid monoclonal immunoglobulin deposition [4, 5]. Moreover, because of the extremely low prevalence of $\mathrm{AH}$ amyloidosis [2-4, 21], it was questioned whether a single patient may develop both $\mathrm{AL}$ and $\mathrm{AH}$ amyloidosis [4]. On the other hand, co-deposition of non-amyloid immunoglobulin with amyloid fibrils is a well-described phenomenon [4-6, 22]. Therefore, we speculate that the present condition might not be so rare and might be more prevalent than "true" AHL amyloidosis.

\section{Conclusion}

We demonstrated "AL amyloidosis with non-amyloid forming monoclonal Ig deposition" disguised as AHL amyloidosis. The confident exclusion of the present condition should be required to diagnose AHL amyloidosis.

\section{Concise method for the two LC-MS/MS analyses}

LMD [2, 3, 18]: Formalin-fixed paraffin-embedded sections were stained with congo-red dye, and the positive areas were extracted using an LMD system (LMD 7000; Leica Microsystems Inc., Tokyo, Japan). The extraction was solubilised in $10 \mathrm{mM}$ Tris/1 mM EDTA/0.002\% Zwittergent buffer and digested overnight with trypsin.

Amyloid purification [7, 14-17]: Fresh frozen renal tissue was homogenised in Tris-buffered saline, after which it was centrifuged and the supernatant was decanted. The procedure was repeated twice. The resultant pellet was solubilised in $6 \mathrm{M}$ guanidine/ $0.5 \mathrm{M}$ Tris-buffered saline, after which it was centrifuged and the supernatant was dialysed against distilled water. The sample was solubilised in gel loading buffer containing 5\% 2-mercaptoethanol and subjected to sodium dodecyl sulfate polyacrylamide gel electrophoresis. The entire electrophoresed sample was excited and digested overnight with trypsin.

The samples were analysed by LC-MS/MS (Nano LC DiNa; KYA Technologies Co., Tokyo, Japan; and QExactive; Thermo Fisher Scientific Inc., Waltham, MA).

\section{Abbreviations \\ $\mathrm{AH}$ amyloidosis: Immunoglobulin heavy-chain amyloidosis; $\mathrm{AHL}$ amyloidosis: Immunoglobulin heavy-and-light-chain amyloidosis; AL amyloidosis: Immunoglobulin light-chain amyloidosis; Ig: Immunoglobulin; LC-MS/MS: Liquid chromatography-tandem mass spectrometry; LMD: Laser microdissection; UPCR: Urinary protein-to-creatinine ratio}

Availability of data and materials

Anonymized data can be provided for reasonable request.

\section{Authors' contributions}

SM designed and drafted the manuscript. $\mathrm{Cl}$ and $\mathrm{MH}$ participate in the interpretation of patient data. FK and MY performed mass spectrometry. KN and NM reviewed the manuscript. All authors read and approved the final manuscript.

\section{Ethics approval and consent to participate}

This study was granted an exemption from requiring ethics approval from research ethics committee of Yokohama Rosai Hospital because this is a case report on an already described treatment and secondary analysis.

\section{Consent for publication}

Written informed consent was obtained from the patient for publication of this case report and any accompanying images.

\section{Competing interests}

The authors declare that they have no competing interests.

\section{Publisher's Note}

Springer Nature remains neutral with regard to jurisdictional claims in published maps and institutional affiliations.

\section{Author details}

${ }^{1}$ Kidney and Vascular Pathology, Faculty of Medicine, University of Tsukuba, 1-1-1 Tennodai, Tsukuba, Ibaraki 305-8575, Japan. ${ }^{2}$ Department of Nephrology, Yokohama Rosai Hospital, Yokohama, Kanagawa, Japan. ${ }^{3}$ Department of Medicine, Kidney Center, Tokyo Women's Medical University, Tokyo, Japan. ${ }^{4}$ Department of Dementia and Higher Brain Function, Tokyo Metropolitan Institute of Medical Science, Tokyo, Japan. ${ }^{5}$ Institute for Biomedical Sciences, Interdisciplinary Cluster for Cutting Edge Research, Shinshu University, Nagano, Japan. 
Received: 22 May 2017 Accepted: 20 September 2018

Published online: 22 November 2018

\section{References}

1. Bridoux F, Leung N, Hutchison CA, Touchard G, Sethi S, Fermand JP, et al. Diagnosis of monoclonal gammopathy of renal significance. Kidney Int. 2015;87(4):698-711.

2. Sethi $\mathrm{S}$, Theis JD, Leung N, Dispenzieri A, Nasr SH, Fidler ME, et al. Mass spectrometry-based proteomic diagnosis of renal immunoglobulin heavy chain amyloidosis. Clin J Am Soc Nephrol. 2010;5(12):2180-7.

3. Nasr SH, Said SM, Valeri AM, Sethi S, Fidler ME, Cornell LD, et al. The diagnosis and characteristics of renal heavy-chain and heavy/light-chain amyloidosis and their comparison with renal light-chain amyloidosis. Kidney Int. 2013;83(3):463-70.

4. Picken MM. Non-light-chain immunoglobulin amyloidosis: time to expand or refine the spectrum to include light+heavy chain amyloidosis? Kidney Int. 2013:83(3):353-6.

5. Kourelis TV, Dasari S, Theis JD, Ramirez-Alvarado M, Kurtin PJ, Gertz MA, et al. Clarifying immunoglobulin gene usage in systemic and localized immunoglobulin light-chain amyloidosis by mass spectrometry. Blood. 2017; 129(3):299-306.

6. Picken MM, Herrera GA. The burden of "sticky" amyloid: typing challenges. Arch Pathol Lab Med. 2007;131(6):850-1.

7. Benson MD, James S, Scott K, Liepnieks JJ, Kluve-Beckerman B. Leukocyte chemotactic factor 2: a novel renal amyloid protein. Kidney Int. 2008;74(2):218-22.

8. Ramirez-Alvarado M. Amyloid formation in light chain amyloidosis. Curr Top Med Chem. 2012;12(22):2523-33.

9. Eulitz M, Weiss DT, Solomon A. Immunoglobulin heavy-chain-associated amyloidosis. Proc Natl Acad Sci U S A. 1990;87(17):6542-6.

10. Solomon A, Weiss DT, Murphy C. Primary amyloidosis associated with a novel heavy-chain fragment (AH amyloidosis). Am J Hematol. 1994;45(2):171-6.

11. Nasr SH, Lobritto SJ, Lauring BP, Arend $\sqcup$, D'Agati VD, Markowitz GS. A rare complication of monoclonal gammopathy. Am J Kidney Dis. 2002;40(4):867-71.

12. Mai HL, Sheikh-Hamad D, Herrera GA, Gu X, Truong LD. Immunoglobulin heavy chain can be amyloidogenic: morphologic characterization including immunoelectron microscopy. Am J Surg Pathol. 2003;27(4):541-5.

13. Copeland JN, Kouides PA, Grieff M, Nadasdy T. Metachronous development of nonamyloidogenic lambda light chain deposition disease and lgG heavy chain amyloidosis in the same patient. Am J Surg Pathol. 2003;27(11):1477-82.

14. Yazaki M, Fushimi T, Tokuda T, Kametani F, Yamamoto K, Matsuda M, et al. A patient with severe renal amyloidosis associated with an immunoglobulin gamma-heavy chain fragment. Am J Kidney Dis. 2004;43(5):e23-8.

15. Gono T, Yazaki M, Fushimi T, Suzuki T, Uehara T, Sano K, et al. AH amyloidosis associated with lymphoplasmacytic lymphoma secreting a monoclonal gamma heavy chain carrying an unusual truncated D segment. Am J Kidney Dis. 2006;47(5):908-14.

16. Miyazaki D, Yazaki M, Gono T, Kametani F, Tsuchiya A, Matsuda M, et al. AH amyloidosis associated with an immunoglobulin heavy chain variable region (VH1) fragment: a case report. Amyloid. 2008;15(2):125-8.

17. Manabe S, Hatano M, Yazaki M, Nitta K, Nagata M. Renal AH amyloidosis associated with a truncated immunoglobulin heavy chain undetectable by Immunostaining. Am J Kidney Dis. 2015;66(6):1095-100.

18. Gallo G, Picken M, Frangione B, Buxbaum J. Nonamyloidotic monoclona immunoglobulin deposits lack amyloid P component. Mod Pathol. 1988; 1(6):453-6.

19. Kinoshita K, Yamagata T, Nozaki Y, Sugiyama M, Ikoma S, Funauchi M, et al Mu-heavy chain disease associated with systemic amyloidosis. Hematology. 2004;9(2):135-7

20. Bianchi G, Anderson KC, Harris NL, Sohani AR. The heavy chain diseases: clinical and pathologic features. Oncology (Williston Park). 2014;28(1):45-53.

21. Said SM, Sethi S, Valeri AM, Leung N, Cornell LD, Fidler ME, et al. Renal amyloidosis: origin and clinicopathologic correlations of 474 recent cases. Clin J Am Soc Nephrol. 2013;8(9):1515-23.

22. Chauvet $S$, Bridoux F, Ecotière L, Javaugue V, Sirac C, Arnulf B, et al. Kidney diseases associated with monoclonal immunoglobulin M-secreting B-cell lymphoproliferative disorders: a case series of 35 patients. Am J Kidney Dis. 2015;66(5):756-67.

\section{Ready to submit your research? Choose BMC and benefit from:}

- fast, convenient online submission

- thorough peer review by experienced researchers in your field

- rapid publication on acceptance

- support for research data, including large and complex data types

- gold Open Access which fosters wider collaboration and increased citations

- maximum visibility for your research: over $100 \mathrm{M}$ website views per year

At BMC, research is always in progress.

Learn more biomedcentral.com/submissions 